\title{
A Variabilidade Climática e os Desastres Naturais no Estado do Ceará (1991-2019)
}

\author{
Marcos Aurélio da Silva Lima ${ }^{1}$ (D), Marcos Antônio Tavares Lira ${ }^{2}$ \\ ${ }^{1}$ Universidade Estadual do Ceará, Fortaleza, CE, Brasil. \\ ${ }^{2}$ Universidade Federal do Piauí, Teresina, PI, Brasil.
}

Recebido em: 16 de Abril de 2021 - Aceito em: 28 de Abril de 2021

\begin{abstract}
Resumo
O objetivo geral da pesquisa foi discutir como os desastres naturais identificados no Estado do Ceará se relacionam com a variabilidade climática, destacando-se aqueles associados à precipitação, no período de 1991 a 2019. No que alude à metodologia, adotou-se pesquisa descritiva, explicativa e de natureza qualitativa-quantitativa. Tratou-se de um estudo de caso, cuja técnica de coletas de dados partiu de pesquisa documental e bibliográfica. A análise de dados foi feita a partir da estatística multivariada, por meio de regressão não-linear e coeficiente de correlação de Pearson. Os dados climáticos foram obtidos, principalmente, a partir da Fundação Cearense de Meteorologia e Recursos Hídricos (FUNCEME), já os registros de desastres, através da Coordenadoria Estadual de Defesa Civil do Ceará (CEDEC-CE) e Universidade Federal de Santa Catarina (UFSC). Os resultados obtidos mostraram que os desastres naturais com maior número de registros foram as estiagens e secas, correlacionando-se de forma alta e negativa com as chuvas, associandose ao fenômeno El Niño e/ou às condições oceano-atmosféricas desfavoráveis às chuvas do Atlântico tropical. Já as inundações, as enxurradas, os alagamentos e as chuvas intensas constituíram o segundo grupo de tipificações de desastres examinados, correlacionando-se de forma moderada e positiva com as chuvas, associando-se ao fenômeno La Niña e/ou às condições oceano-atmosféricas favoráveis às chuvas do Atlântico tropical. Logo, concluiu-se que há uma tendência de aumento do número de desastres naturais no Ceará, os quais estão relacionados com a variabilidade climática.
\end{abstract}

Palavras-chave: climatologia, mudanças climáticas, defesa civil.

\section{Climate Variability and Natural Disasters in the State of Ceará (1991-2019)}

\begin{abstract}
The general objective of the research was to discuss how the natural disasters identified in the State of Ceará are related to climatic variability, highlighting those associated with precipitation, in the period from 1991 to 2019. Regarding the methodology, descriptive research was adopted, explanatory and qualitative-quantitative in nature. It was a case study, whose data collection technique started from documentary and bibliographic research. Data analysis was performed using multivariate statistics, using non-linear regression and Pearson's correlation coefficient. The climatic data were obtained, mainly, from the Cearense Foundation of Meteorology and Water Resources (FUNCEME), already the records of disasters, through the State Coordination of Civil Defense of Ceará (CEDEC-CE) and the Federal University of Santa Catarina (UFSC). The results obtained showed that the natural disasters with the greatest number of records were droughts and droughts, correlating highly and negatively with the rains, being associated with the El Niño phenomenon and / or the ocean-atmospheric conditions unfavorable to the rains of the Tropical Atlantic. Floods, floods, floods and heavy rains were the second group of types of disasters examined, correlating moderately and positively with rainfall, associating with the La Niña phenomenon and / or ocean-atmospheric conditions. favorable to tropical Atlantic rains. Therefore, it was concluded that there is a tendency to increase the number of natural disasters in Ceará, which are related to climatic variability.
\end{abstract}

Keywords: climatology, climate changes, civil defense.

Autor de correspondência: Marcos Aurélio da Silva Lima, marcos.aureliolima@gmail.com. 


\section{Introdução}

Em seu estudo sobre a atmosfera, o tempo e o clima, Barry e Chorley (2013, p. 392) afirmam que o mais importante trabalho realizado na segunda metade do século XX foi o reconhecimento da existência do sistema climático global. Nessa perspectiva, aduzem que "os fenômenos meteorológicos abrangem uma ampla variedade de escalas espaciais e temporais, desde rajadas de vento, que levantam folhas e outros detritos, aos sistemas de ventos em escala global, que moldam o clima planetário."

Isso posto, tem-se que a mudança do clima, associada ao aquecimento global, pode alterar frequência, intensidade, distribuição espacial, duração, sincronismo dos eventos extremos, e resultar em condições excepcionais, até mesmo nunca antes observadas (IPCC, 2014).

Conforme Goerl e Kobiyama (2013), a propósito, alguns setores da sociedade e até pesquisadores mencionam, inclusive, um aumento na frequência de desastres naturais nas últimas décadas, com destaque para aqueles que estão relacionados à precipitação.

Situado na Região Nordeste do Brasil (NEB), o Estado do Ceará sofre tanto com as secas e estiagens, como também pela ocorrência de eventos extremos, associados às precipitações elevadas, sendo tipificados como inundações, enxurradas e alagamentos, os quais podem ser correlacionados à atuação de diversos sistemas atmosféricos e fenômenos climáticos.

No Brasil, é papel da Defesa Civil a oficialização do registro de um desastre - seja tecnológico ou natural -, que pode ocorrer pela decretação municipal ou estadual de situação de emergência ou estado de calamidade pública, com reconhecimento federal.

Tendo isso em vista, concebe-se a necessidade de prescrutar as informações referentes ao estado do Ceará em busca dos dados catalogados por sua CEDEC-CE, a fim de verificar o comportamento dos registros de desastres naturais nos últimos 30 anos.

Nesse ensejo, o problema da pesquisa incide na busca por resposta para a seguinte indagação: como os desastres naturais identificados no Estado do Ceará estão relacionados à variabilidade climática, com destaque aos associados à precipitação?

Com o propósito de responder a tal indagação, esta investigação parte de uma pesquisa descritiva, explicativa e de natureza qualitativa-quantitativa. Trata-se, pois, de um estudo de caso, cuja coletas de dados se dá a partir de pesquisa documental e bibliográfica, com análise de dados de conteúdo mediante estatística multivariada.

O objetivo geral da pesquisa é discutir como os desastres naturais identificados no Estado do Ceará se relacionam com a variabilidade climática, destacando-se aqueles associados à precipitação, no período de 1991 a 2019.

Os objetivos específicos são: analisar a variabilidade climática no estado do Ceará, principalmente quanto à precipitação associada aos sistemas atmosféricos e fenômenos climáticos, no período de 1991 a 2019; identificar e classificar os desastres naturais registrados no estado do Ceará, no período de 1991 a 2019; correlacionar estatisticamente os desastres naturais identificados no Estado do Ceará com a variabilidade climática, sobretudo quanto à precipitação, no período de 1991 a 2019.

\section{Metodologia}

$\mathrm{Na}$ pesquisa quantitativa, coletaram-se os registros oficiais de desastres naturais e os dados das precipitações médias normais no período de estudo, de 1991 a 2019, nos 184 municípios do estado de Ceará. Todas as informações foram extraídas principalmente dos seguintes bancos de dados: da FUNCEME, Instituto Nacional de Meteorologia (INMET), Instituto Nacional de Pesquisas Espaciais (INPE), CEDEC-CE, UFSC e Sistema Integrado de Informações sobre Desastres (S2ID).

De forma geral, cumpriu-se uma análise da variabilidade climática, dos desastres naturais e a sua interrelação conforme ilustra a Fig. 1.

Então, os resultados indicarão correlação forte ou fraca entre as seguintes varáveis $\mathrm{X}$ e $\mathrm{Y}$, as quais se deseja

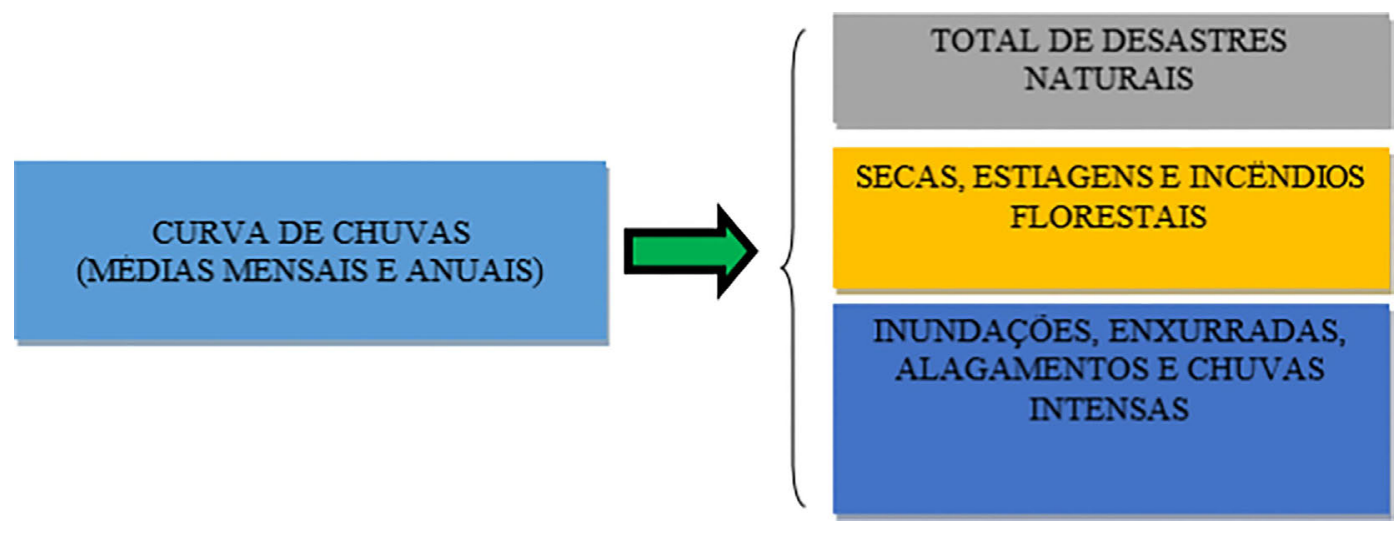

Figura 1 - Etapas da análise da pesquisa de variabilidade x desastres naturais. Fonte: Lima (2020). 
verificar a existência de correlação, no caso: $\mathrm{X}=$ quantidade de chuvas e $\mathrm{Y}=$ quantidade de desastres naturais. Além disso, serão obtidos o polinômio da análise regressão não-linear e construídos gráficos comparativos.

A partir do software Geogebra, denotou-se a possibilidade de modelar a relação entre as variáveis da pesquisa, utilizando-se da análise de regressão não-linear polinomial. O estudo possibilitou o reconhecimento do comportamento de uma grandeza independente em função da outra dependente. As séries são comparadas e apresentam-se os diagramas de dispersão.

Ainda através do Geogebra, foi feita a análise de regressão não-linear (polinômio com equação de nono grau) e obtidas as equações: da média $(\bar{x})$, do desvio padrão $(S)$, do coeficiente de correlação de Pearson $(r)$, do coeficiente de covariância $(\rho)$ e do coeficiente de representação de regressão linear $\left(R^{2}\right)$, conforme Eqs. (1)-(5).

Média :

$$
\bar{x}=\frac{1}{n} \sum_{i=1}^{n} x i
$$

Desvio padrão :

$$
S=\sqrt{\frac{\sum_{i=1}^{K}\left(x_{i}-x\right)^{2} f_{i}}{n-1}}
$$

Coeficiente de correlação de Pearson :

$$
r=\frac{\sum\left(x_{i}-\bar{x}\right)\left(y_{i}-\bar{y}\right)}{\sqrt{\sum\left(\left(x_{i}-\bar{x}\right)^{2}\right) \cdot\left(\sum\left(y_{i}-\bar{y}\right)^{2}\right)}}
$$

Covariância :

$$
\rho=\frac{\sum\left(x_{i}-\bar{x}\right)\left(y_{i}-\bar{y}\right)}{(n-1)}
$$

Coeficiente de determinação $R^{2}$ :

$$
R^{2}=\left(\frac{\left.\sum\left(x_{i}-\bar{x}\right)\left(y_{i}-\bar{y}\right)\right)^{2}}{\sum\left(\left(x_{i}-\bar{x}\right)^{2}\right) \cdot\left(\sum\left(y_{i}-\bar{y}\right)^{2}\right)}\right.
$$

Os valores dos coeficientes de correlação de Pearson foram interpretados de acordo com o modelo proposto por Bisquerra et al. (2004), como mostra o Tabela 1. Para os coeficientes negativos, houve a mesma interpretação.

Finalmente, a partir das séries temporais das varáveis supracitadas, assimilou-se também a possibilidade de visualização de sazonalidade, ciclos e tendências.
Tabela 1 - Interpretação do valor do coeficiente de Pearson.

\begin{tabular}{lc}
\hline Coeficiente & Interpretação \\
\hline $\mathrm{r}=1$ & Correlação perfeita \\
$0,8<\mathrm{r}<1$ & Muito alta \\
$0,6<\mathrm{r}<0,79$ & Alta \\
$0,4<\mathrm{r}<0,59$ & Moderada \\
$0,2<\mathrm{r}<0,39$ & Baixa \\
$0<\mathrm{r}<0,19$ & Muito baixa \\
$\mathrm{r}=0$ & Nula \\
\hline
\end{tabular}

Fonte: Bisquerra et al. (2004).

\section{Resultados e Discussões}

De acordo com Machado (2017), o clima é a estatística das variáveis que definem o estado da atmosfera dentro de um período de tempo. Essas variáveis são: temperatura; pressão; vento (direção e intensidade); umidade do ar; radiação solar e precipitação.

A partir das informações do INMET (2020) e Funceme (2020), obtiveram-se os valores de cada uma das variáveis mensais da série histórica mais recente (19812010), a partir dos postos de coleta de dados dos municípios cearenses a seguir: Acaraú, Barbalha, Campos Sales, Crateús, Fortaleza, Guaramiranga, Iguatu, Jaguaruana, Morada Nova, Quixeramobim, Sobral e Tauá.

Em ênfase, a variável climatológica com maior variabilidade de dados na série temporal mensal foi a precipitação pluviométrica, conforme Fig. 2.

$\mathrm{Na}$ Fig. 3. destacam-se os valores máximos acumulados de chuvas durante a pré-estação, em janeiro, e na própria estação chuvosa, de fevereiro a maio.

De toda forma, o regime de chuvas sofre variações não somente ao longo de um ano, mas também entre anos, tendo como resultado uma variabilidade de chuvas anuais que dependem ou sofrem influência de sistemas atmosféricos e fenômenos climáticos de maior intensidade e escala, a exemplo da Zona de Convergência Intertropical (ZCIT), do fenômeno El Niño Oscilação Sul (ENOS) e do Dipolo, a partir da Temperatura da Superfície do Mar (TSM) do Atlântico Tropical - vide oscilação das médias pluviométricas no Ceará, na série temporal de estudo, em relação ao valor normal de chuvas, construiu-se a Fig. 3.

A variabilidade climática, visualizada nas Figs. 2 e 3, pode ser explicada a partir da atuação de diversos sistemas atmosféricos e fenômenos climáticos, como: ENOS; TSM do Oceano Atlântico tropical e ZCIT, Frentes Frias (FF), Vórtice Ciclônico de Altos Níveis (VCAN), Linha de Instabilidade (LI), Zona Frontal (ZF), Complexos Convectivos de Mesoescala (CCM), Zona de Convergência do Attântico Sul (ZCAS), Ondas de leste (OL), Distúrbios Ondulatórios de Leste (DOL), Anticiclone Subtropical do Atlântico Sul (ASAS), Vórtice Ciclônico de Ar Superior (VCAS) e Brisa. 


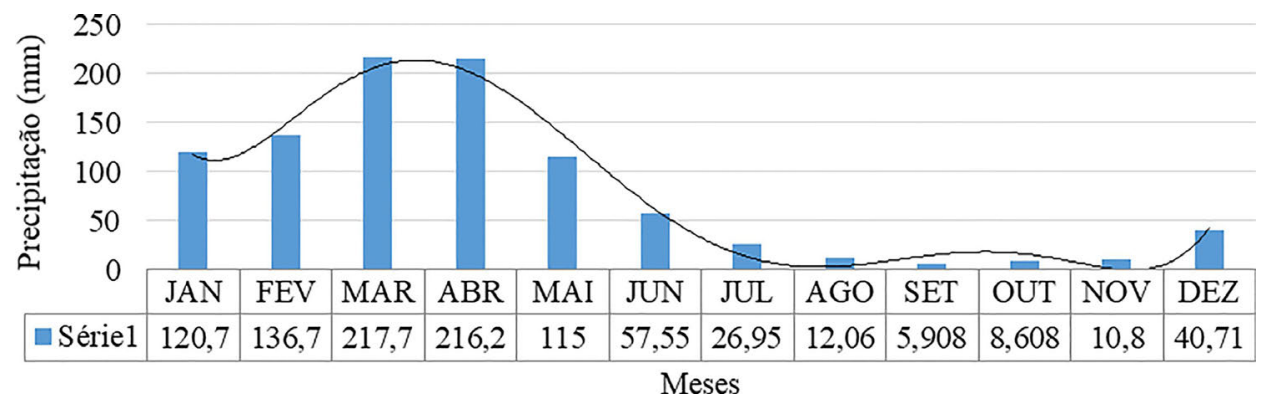

Figura 2 - Precipitações mensais médias (mm) no Ceará de 1980-2010. Fonte: Lima (2020), INMET (2020) e Funceme (2020).

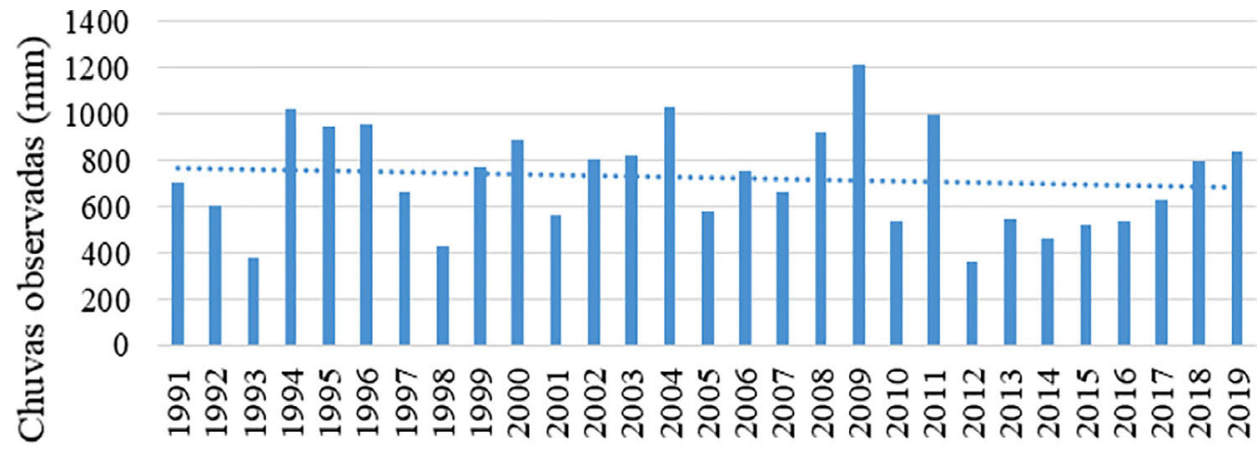

Ano

Figura 3 - Médias pluviométricas no Ceará (1991-2019). Fonte: Funceme (2020).

Em consonância com o período de ocorrência das chuvas no ano, concebe-se uma classificação pela meteorologia, com a indicação dos principais sistemas atmosféricos atuantes no Ceará, como dispõe a Tabela 2.

Já o total de registros anuais de desastres naturais coletados no Estado do Ceará foi compilado conforme mostra a Fig. 4.

Com o total de 3782 registros, houve a identificação de dez tipos de desastres naturais no estado do Ceará de 1991 a 2019, a saber: secas; estiagens; enxurradas; inundações; alagamentos; erosão; incêndios florestais; movimentos de massa; vendavais; e chuvas intensas.

No estudo, observa-se que nos anos de 1991, 1992 e de 1994 a 1998, não há dados de registros de desastres no Ceará, pois na pesquisa pioneira sobre dados de desastres

Tabela 2 - Resumo dos principais desastres naturais relacionados aos sistemas atmosféricos e anomalias climáticas atuantes no Ceará, por período do ano (1991-2019).

\begin{tabular}{lcc}
\hline $\begin{array}{l}\text { Período de } \\
\text { ocorrência }\end{array}$ & $\begin{array}{c}\text { Classificação pela } \\
\text { meteorologia }\end{array}$ & $\begin{array}{c}\text { Principais sistemas } \\
\text { atmosféricos }\end{array}$ \\
\hline $\begin{array}{l}\text { dezembro a } \\
\text { janeiro } \\
\text { fevereiro a maio }\end{array}$ & Chuvas de pré-estação & $\begin{array}{c}\text { FF, VCAN, LI, ZF, CCM, } \\
\text { ZCAS, Brisa }\end{array}$ \\
$\begin{array}{l}\text { junho a } \\
\text { novembro ou quadra } \\
\text { chuvosa }\end{array}$ & ZCIT, LI, Brisa \\
\hline Chuvas de pós-estação & $\begin{array}{c}\text { OL, DOL, LI, ZF, ASAS, } \\
\text { VCAS e Brisa }\end{array}$ \\
\hline
\end{tabular}

Fonte: Lima (2020). naturais no Brasil, a saber, a construção do Atlas Brasileiro de Desastres Naturais, a equipe responsável pela coleta de informações junto aos agentes de defesa civil não encontraram documentos oficiais no arquivo da CEDECCE referentes aos anos citados.

Os registros dos desastres naturais mais recorrentes foram distribuídos em uma frequência mensal ao longo dos anos de 1991 a 2019 - vide Fig. 5.

Analisando-se ainda a Fig. 5, é possível identificar que os desastres relativos ao excesso de chuvas, como inundações, enxurradas e alagamentos, concentram-se nos meses do período chuvoso do estado - novembro a maio - apresentando maior frequência mensal de janeiro a maio. De outra forma, os desastres por estiagens e secas distribuem-se por todos os meses do ano. No entanto, a maior frequência do evento está nos meses do período de pouca chuva no estado, entre junho e outubro, com picos em maio - com o fim da quadra chuvosa característica da região - e novembro - que marca o início das chuvas de pré-estação chuvosa.

Para fins de apresentação nesta pesquisa, definiu-se que os registros de secas e estiagens comporiam o mesmo grupo quantitativo intitulado secas e estiagens.

Na Tabela 3, mostra-se o total de desastres naturais, classificados por tipo, no período de 1991 a 2019.

Para mais, as estiagens e secas tiveram registros de ocorrências em quase todos os anos, sobrelevando-se o recente período de 2012 a 2017, conforme Tabela 4. 


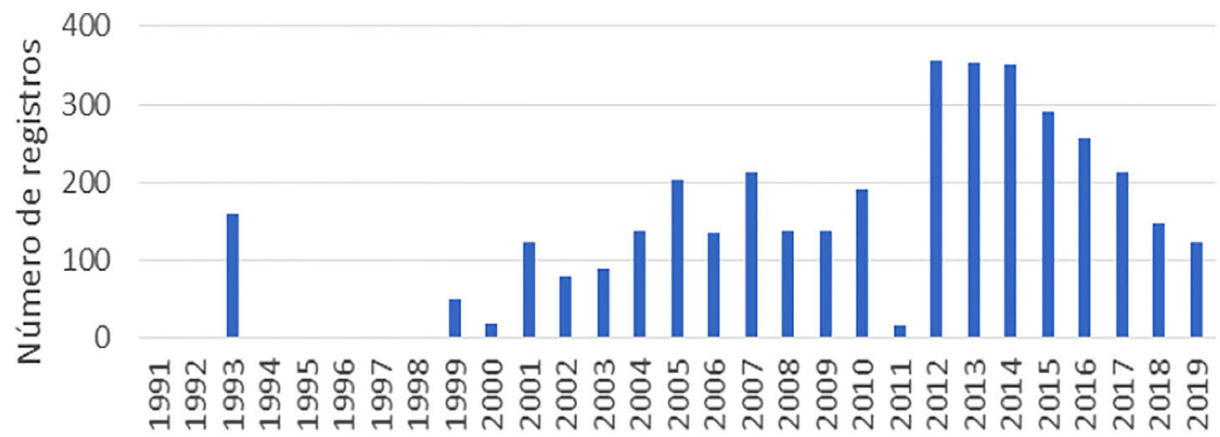

Ano

Figura 4 - Registros oficiais de desastres naturais no Ceará (1991-2019). Fonte: Lima (2020), UFSC (2013) e Cedec/CE (2020).

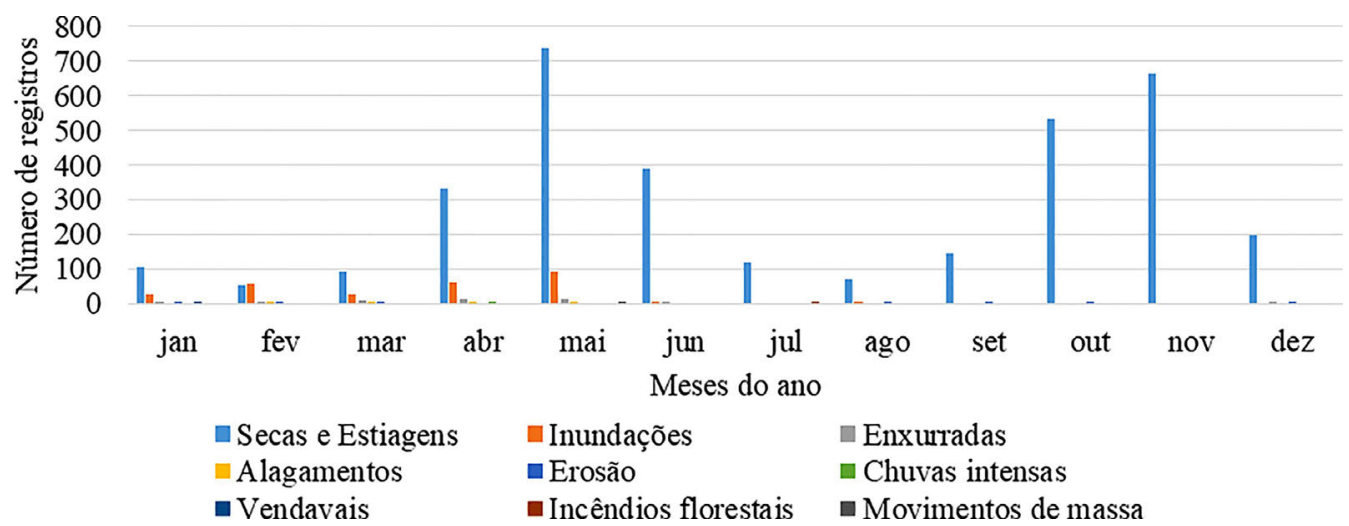

Figura 5 - Frequência mensal de desastres naturais no Ceará (1991-2019). Fonte: Lima (2020), UFSC (2013) e Cedec/CE (2020).

A Fig. 6 mostra todos os registros de desastres por município e mesorregião do estado do Ceará. Todos os 184 municípios foram atingidos por algum tipo de evento de 1991 a 2019, destacando-se o maior quantitativo na mesoregião Sertões Cearenses. Os municípios de Caridade, Catunda, Caucaia, Madalena, Mombaça, Parambu, Pedra Branca, Quixeramobim, Tabuleiro do Norte e Tauá

Tabela 3 - Quantidade de desastres naturais registrados no Ceará de 1991 a 2019.

\begin{tabular}{lcc}
\hline Tipo de desastre natural & Número de registros & Valores percentuais (\%) \\
\hline Secas e Estiagem & 3438 & 90,90 \\
Inundações & 273 & 7,22 \\
Enxurradas & 51 & 1,35 \\
Erosões & 10 & 0,26 \\
Alagamentos & 6 & 0,16 \\
Vendavais & 1 & 0,03 \\
Movimentos de massa & 1 & 0,03 \\
Incêndios florestais & 1 & 0,03 \\
Chuvas intensas & 1 & 0,03 \\
Total & 3782 & 100,00 \\
\hline
\end{tabular}

Fonte: Lima (2020), UFSC (2013) e Cedec/CE (2020). apresentam a maior frequência de desastres, dispostos na classe de 30-37 ocorrências.

Nessa perspectiva, foi realizado o estudo da correlação entre os desastres naturais e a precipitação pluviométrica no estado do Ceará (1991-2019), com ênfase nos tipos de desastres naturais que possuem uma maior relação com o regime de chuvas do Ceará, a saber: secas; estiagens; incêndios florestais; inundações; enxurradas; alagamentos e chuvas intensas.

Portanto, a priori, pretende-se efetivar o estudo comparativo da média mensal de chuvas com o histórico mensal de registros de desastres naturais, vide Fig. 7.

Foi feita a análise multivariada no software Geogebra, obtendo-se o diagrama de dispersão ilustrado na Fig. 8 .

A Eq. (6) representa a equação de regressão não-linear (polinômio com equação de nono grau), dos desastres naturais em função do volume de chuvas mensais.

$$
\begin{gathered}
y=f(x)=1,7575 \mathrm{E}-13 \times 9-1,5183 \mathrm{E}-10 \times 8+ \\
5,3709 \mathrm{E}-8 \times 7-1,0101 \mathrm{E}-5 \times 6+1,1008 \mathrm{E}-3 \times 5- \\
0,0712 \times 4+2,6986 \times 3-56,067 \times 2+ \\
548,92 \times-1541,6
\end{gathered}
$$


Tabela 4 - Quantidade de desastres naturais registrados no Ceará de 1991 a 2019, por tipo e ano.

\begin{tabular}{|c|c|c|c|c|c|c|c|c|c|c|}
\hline Ano & $\begin{array}{l}\text { Estiagens e } \\
\text { secas }\end{array}$ & Inundações & Enxurradas & Erosões & Alagamentos & $\begin{array}{l}\text { Chuvas inten- } \\
\text { sas }\end{array}$ & $\begin{array}{l}\text { Movimento de } \\
\text { massa }\end{array}$ & Vendavais & $\begin{array}{l}\text { Incêndios flor- } \\
\text { estais }\end{array}$ & Total \\
\hline 1991 & 0 & 0 & 0 & 0 & 0 & 0 & 0 & 0 & 0 & 0 \\
\hline 1992 & 0 & 0 & 0 & 0 & 0 & 0 & 0 & 0 & 0 & 0 \\
\hline 1993 & 160 & 0 & 0 & 0 & 0 & 0 & 0 & 0 & 0 & 160 \\
\hline 1994 & 0 & 0 & 0 & 0 & 0 & 0 & 0 & 0 & 0 & 0 \\
\hline 1995 & 0 & 0 & 0 & 0 & 0 & 0 & 0 & 0 & 0 & 0 \\
\hline 1996 & 0 & 0 & 0 & 0 & 0 & 0 & 0 & 0 & 0 & 0 \\
\hline 1997 & 0 & 0 & 0 & 0 & 0 & 0 & 0 & 0 & 0 & 0 \\
\hline 1998 & 0 & 0 & 0 & 0 & 0 & 0 & 0 & 0 & 0 & 0 \\
\hline 1999 & 49 & 0 & 0 & 0 & 0 & 0 & 0 & 0 & 0 & 49 \\
\hline 2000 & 16 & 0 & 3 & 0 & 0 & 0 & 0 & 0 & 0 & 19 \\
\hline 2001 & 113 & 0 & 10 & 0 & 0 & 0 & 0 & 0 & 0 & 123 \\
\hline 2002 & 70 & 2 & 5 & 0 & 0 & 0 & 1 & 0 & 0 & 78 \\
\hline 2003 & 75 & 6 & 6 & 1 & 1 & 0 & 0 & 0 & 0 & 89 \\
\hline 2004 & 42 & 94 & 2 & 0 & 0 & 0 & 0 & 0 & 0 & 138 \\
\hline 2005 & 200 & 1 & 1 & 0 & 0 & 0 & 0 & 1 & 0 & 203 \\
\hline 2006 & 136 & 0 & 0 & 0 & 0 & 0 & 0 & 0 & 0 & 136 \\
\hline 2007 & 211 & 0 & 0 & 1 & 0 & 0 & 0 & 0 & 0 & 212 \\
\hline 2008 & 80 & 56 & 0 & 1 & 1 & 0 & 0 & 0 & 0 & 138 \\
\hline 2009 & 7 & 110 & 17 & 1 & 2 & 0 & 0 & 0 & 0 & 137 \\
\hline 2010 & 189 & 2 & 0 & 1 & 0 & 0 & 0 & 0 & 0 & 192 \\
\hline 2011 & 8 & 2 & 3 & 3 & 0 & 0 & 0 & 0 & 0 & 16 \\
\hline 2012 & 354 & 0 & 1 & 0 & 0 & 0 & 0 & 0 & 1 & 356 \\
\hline 2013 & 351 & 0 & 1 & 1 & 0 & 0 & 0 & 0 & 0 & 353 \\
\hline 2014 & 351 & 0 & 0 & 0 & 0 & 0 & 0 & 0 & 0 & 351 \\
\hline 2015 & 291 & 0 & 0 & 0 & 0 & 0 & 0 & 0 & 0 & 291 \\
\hline 2016 & 257 & 0 & 0 & 0 & 0 & 0 & 0 & 0 & 0 & 257 \\
\hline 2017 & 212 & 0 & 0 & 0 & 0 & 1 & 0 & 0 & 0 & 213 \\
\hline 2018 & 143 & 0 & 1 & 1 & 2 & 0 & 0 & 0 & 0 & 147 \\
\hline 2019 & 123 & 0 & 1 & 0 & 0 & 0 & 0 & 0 & 0 & 124 \\
\hline Total & 3.438 & 273 & 51 & 10 & 6 & 1 & 1 & 1 & 1 & 3782 \\
\hline$\%$ & $90,90 \%$ & $7,22 \%$ & $1,35 \%$ & $0,26 \%$ & $0,16 \%$ & $0,03 \%$ & $0,03 \%$ & $0,03 \%$ & $0,03 \%$ & 1 \\
\hline
\end{tabular}

Fonte: Lima (2020), UFSC (2013) e Cedec/CE (2020).

A Tabela 5 mostra os resultados das variáveis estatísticas obtidos a partir da simulação realizada no software Geogebra e Eqs. (1)-(5).

A interpretação dos resultados, a partir da simulação no software Geogebra, indicou que apesar do valor do coeficiente de determinação $\mathrm{R}^{2}$ ser REPRESENTATIVO, demonstrando que o polinômio de regressão não-linear representou de forma válida os pontos de intersecção das variáveis analisadas, o valor da correlação de Pearson apresentou um valor classificado como MUITO BAIXO e NEGATIVO, evidenciando a FRAQUEZA da relação entre as duas variáveis ao nível de significância menor ou igual a 0,05 .

Em seguida, da mesma forma, promoveu-se o estudo comparativo da média anual de chuvas com o histórico de registros de desastres naturais ao longo da série temporal da pesquisa, de 1991 a 2019, como mostra a Fig. 9.

Observa-se, em geral, que quando há picos de valores acumulados de chuvas, como ocorreu em 1999, 2004, 2009, 2011 e 2019, há diminuição dos registros de desastres naturais. Por outro lado, quando se concebem valores acumulados de chuvas no ano, como em 2001, 2005, 2010, 2012, entre outros, nota-se um considerável aumento do número de registros de desastres naturais.

Foi feita a análise multivariada no software Geogebra, obtendo-se o diagrama de dispersão ilustrado na Fig. 10.

A Eq. (7) representa a equação de regressão não-linear (polinômio com equação de nono grau), dos desastres 


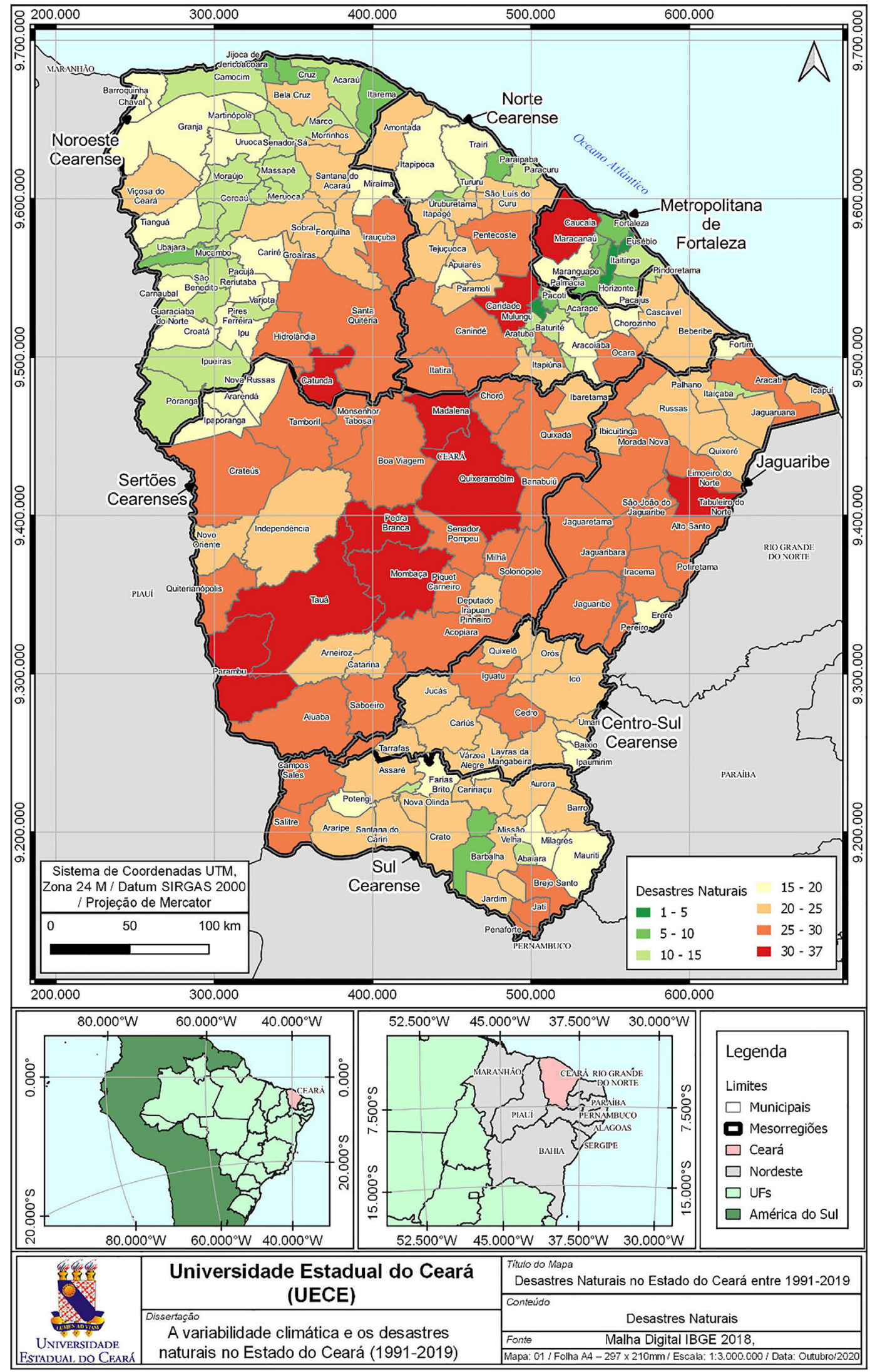

Figura 6 - Municípios com registros oficiais de desastres naturais no Ceará. Fonte: Lima (2020), UFSC (2013) e Cedec/CE (2020). 


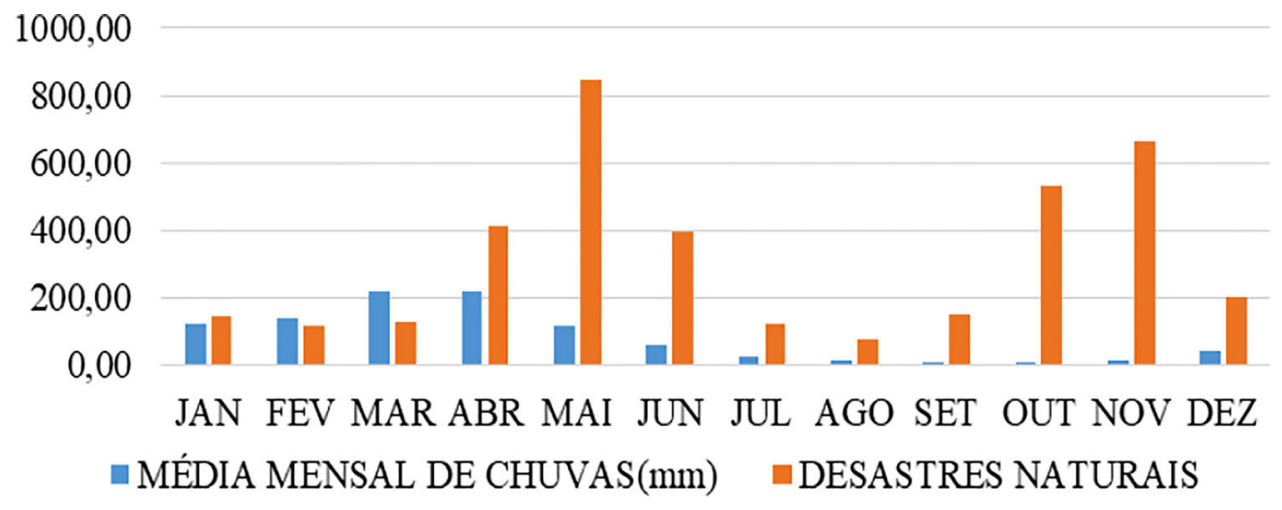

Figura 7 - Acumulado mensal de chuvas x desastres naturais no Ceará (1991-2019). Fonte: Lima (2020), Funceme (2020) e Cedec (2020).

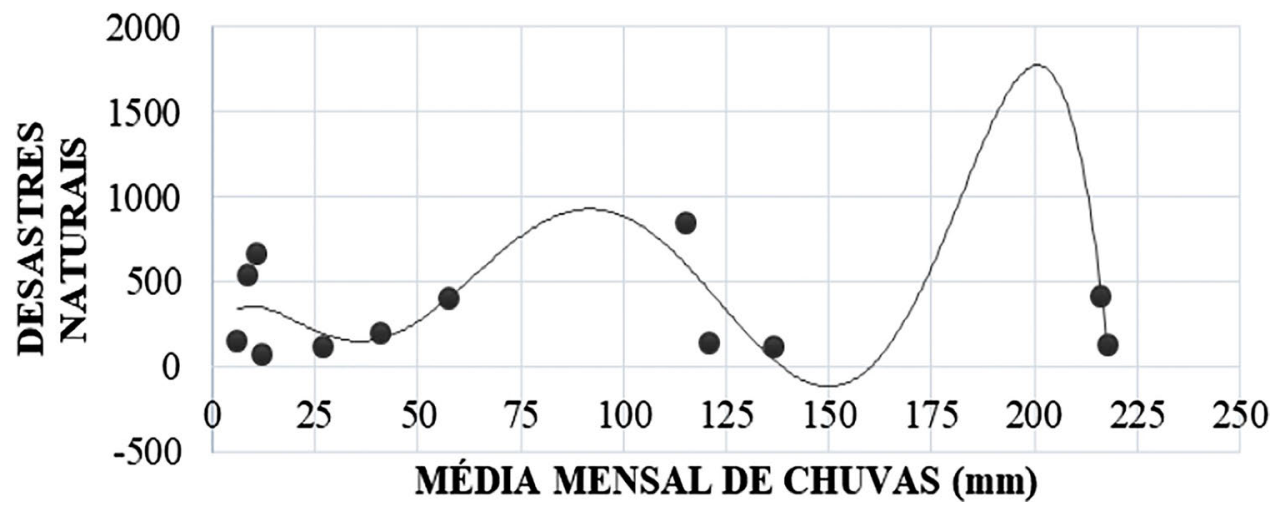

Figura 8 - Acumulado mensal de chuvas x desastres naturais no Ceará (1991-2019). Fonte: Lima (2020).

naturais em função do volume de chuvas anuais, entre 1991 a 2019.

$$
\begin{gathered}
y=f(x)=-4,8929 \mathrm{E}-20 \times 9-3,3501 \mathrm{E}-16 \mathrm{x} 8+ \\
1,0070 \mathrm{E}-12 \times 7-1,7435 \mathrm{E}-12 \times 6+1,9154 \mathrm{E}- \\
6 \times 5-1,3835 \mathrm{E}-3 \times 4-0,6565 \times 3+197,13 \times 2- \\
339,38 \mathrm{x}+2549300
\end{gathered}
$$

Tabela 5 - Resultados da simulação dos dados da média mensal de chuvas e quantidade de desastres naturais registrados no Ceará, de 1991 a 2019, no software Geogebra.

\begin{tabular}{lc}
\hline Variáveis & Resultados \\
\hline Média $(\mathrm{X})$ & 80,743 \\
Média $(\mathrm{Y})$ & 315,58 \\
Desvio padrão de X $\left(\mathrm{S}_{\mathrm{x}}\right)$ & 78,887 \\
Desvio padrão de $\mathrm{Y}\left(\mathrm{S}_{\mathrm{y}}\right)$ & 253,75 \\
Coeficiente de determinação $\left(\mathrm{R}^{2}\right)$ & 0,73003 \\
Coeficiente de correlação de Pearson $(\mathrm{r})$ & $-0,046525$ \\
Coeficiente de correlação, covariância $(\rho)$ & $-0,16084$ \\
SEQ & 191220 \\
\hline
\end{tabular}

Fonte: Lima (2020).
Na Tabela 6, visualizam-se os resultados das variáveis estatísticas a partir da simulação no software Geogebra.

A interpretação dos resultados, a partir da simulação no software Geogebra, indica que o valor do coeficiente de determinação $\mathrm{R}^{2}$ é REPRESENTATIVO, demonstrando que o polinômio de regressão não-linear representou de forma válida os pontos de intersecção das variáveis analisadas e que o valor da correlação de Pearson apresentou um valor classificado como MODERADO e NEGATIVO, revelando a $F O R C ̧ A$ da relação entre as duas variáveis ao nível de significância menor ou igual a 0,05 .

Dessa forma, as variáveis comportaram-se na série temporal de 1991-2019, da seguinte forma: quanto maior o valor observado das chuvas acumuladas, menor o número de registros oficiais de desastres naturais.

Dando seguimento, estabelece-se o estudo comparativo da média anual de chuvas com o histórico de registros de secas, estiagens e incêndios florestais ao longo da série temporal da pesquisa, de 1991 a 2019, como mostra o Fig. 11.

Observa-se, em geral, que quando há picos de valores acumulados de chuvas, como em 1999, 2004, 2009, 2011 e 2019, tem-se uma diminuição dos registros de secas, estiagens e incêndios florestais. Contudo, quando há valores acumulados de chuvas no ano, como em 2001, 


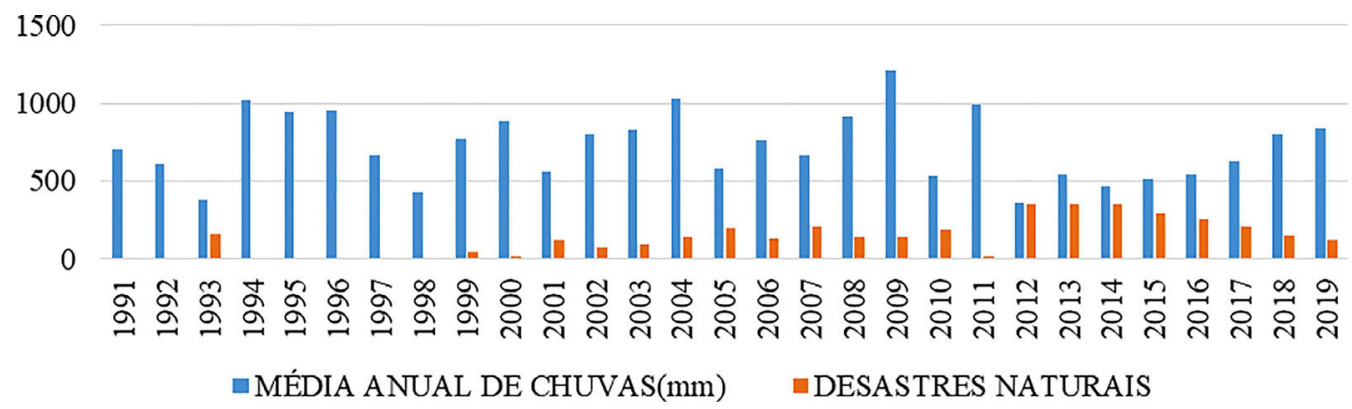

Figura 9 - Acumulado anual de chuvas x desastres naturais no Ceará (1991-2019). Fonte: Lima (2020), Funceme (2020) e Cedec (2020).

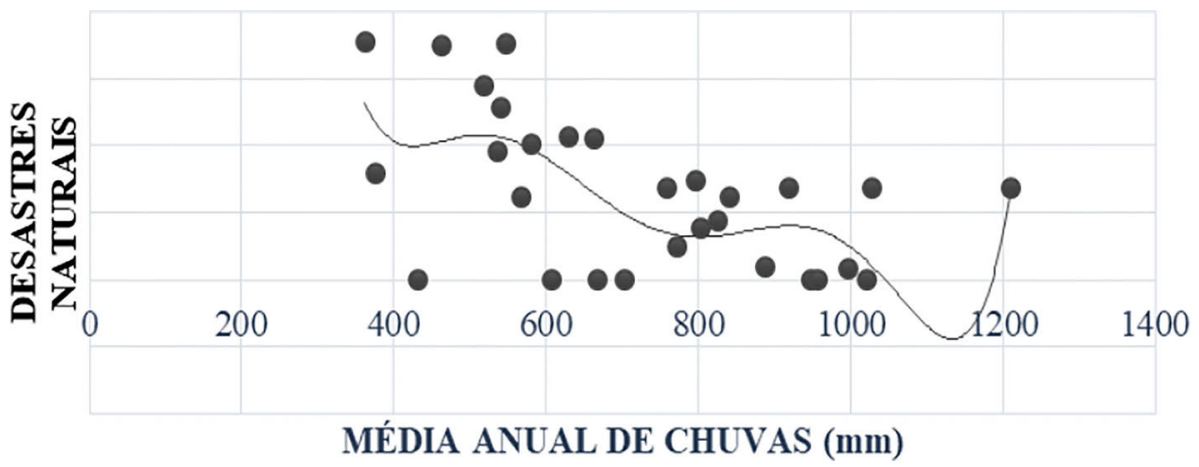

Figura 10 - Relação entre as chuvas e os desastres naturais no Ceará (1991-2019). Fonte: Lima (2020).

2005, 2010, 2012, assiste-se a um considerável aumento do número de registros de tais desastres naturais. É oportuno notar a harmonia dos dados entre os anos de 2013 e 2019 , sendo possível denotar que os desastres registrados são inversamente proporcionais às chuvas acumuladas naqueles anos.

Foi feita a análise multivariada no software Geogebra, obtendo-se o diagrama de dispersão ilustrado na Fig. 12.

A Eq. (8) representa a equação de regressão não-linear (polinômio com equação de nono grau), das secas, estiagens e incêndios florestais em função do volume de

Tabela 6 - Resultados da simulação dos dados da média mensal de chuvas e quantidade de desastres naturais registrados no Ceará de 1991 a 2019, no software Geogebra.

\begin{tabular}{lc}
\hline Variáveis & Resultados \\
\hline Média $(\mathrm{X})$ & 722,91 \\
Média $(\mathrm{Y})$ & 130,41 \\
Desvio padrão de $\mathrm{X}\left(\mathrm{S}_{\mathrm{x}}\right)$ & 217,44 \\
Desvio padrão de $\mathrm{Y}\left(\mathrm{S}_{\mathrm{y}}\right)$ & 115,04 \\
Coeficiente de determinação $\left(\mathrm{R}^{2}\right)$ & 0,62857 \\
Coeficiente de correlação de Pearson $(\mathrm{r})$ & $-0,52549$ \\
Coeficiente de correlação, covariância $(\rho)$ & $-0,51372$ \\
SEQ & 137620 \\
\hline
\end{tabular}

Fonte: Lima (2020). chuvas anuais, entre 1991 a 2019.

$$
\begin{gathered}
y=f(x)=-3,3462 \mathrm{E}-20 \times 9+2,3492 \mathrm{E}-16 \times 8- \\
7,2433 \mathrm{E}-13 \times 7+1,2864 \mathrm{E}-9 \times 6-1,4490 \mathrm{E}- \\
6 \times 5+1,0072 \mathrm{E}-3 \times 4-0,5206 \times 3+159,71 \times 2- \\
28041,81 \mathrm{x}+2143901,671
\end{gathered}
$$

A Tabela 7 evidencia os resultados das variáveis estatísticas a partir da simulação no software Geogebra.

A interpretação dos resultados, a partir da simulação no software Geogebra, indica que o valor do coeficiente de determinação $\mathrm{R}^{2}$ é REPRESENTATIVO, demonstrando que o polinômio de regressão não-linear representou de forma válida os pontos de intersecção das variáveis analisadas e que o valor da correlação de Pearson apresentou um valor classificado como ALTO e NEGATIVO, revelando a $F O R C ̧ A$ da relação entre as duas variáveis ao nível de significância menor ou igual a 0,05 .

Dessa forma, as variáveis comportam-se na série temporal de 1991-2019 da seguinte forma: quanto maior o valor observado das chuvas acumuladas, menor o número de registros oficiais de secas, estiagens e incêndios florestais.

Ainda nessa dinâmica da pesquisa, promove-se o estudo comparativo da média de chuvas com o histórico de registros de inundações, enxurradas, alagamentos e 


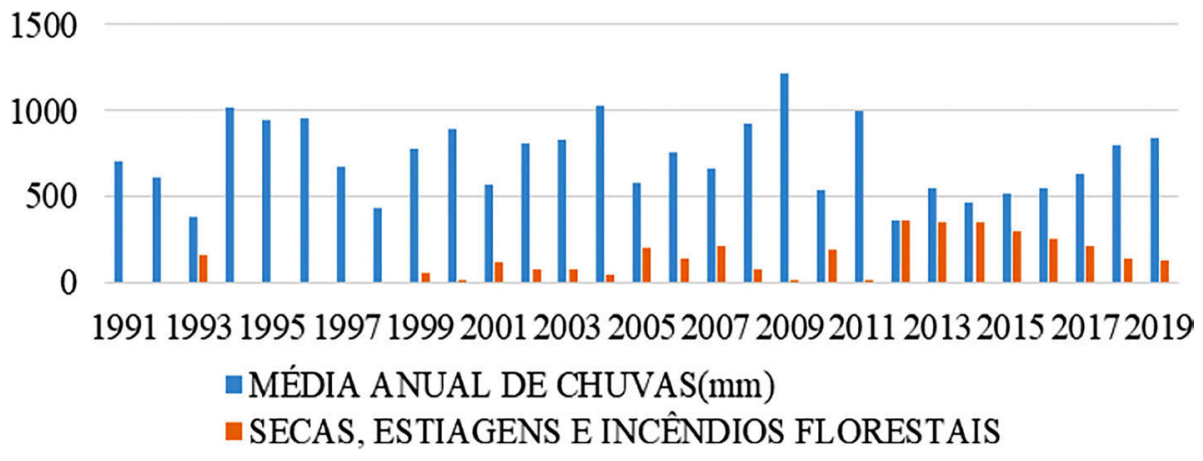

Figura 11 - Acumulado mensal de chuvas x secas, estiagens, incêndios florestais no Ceará (1991-2019). Fonte: Lima (2020), Funceme (2020) e Cedec (2020).

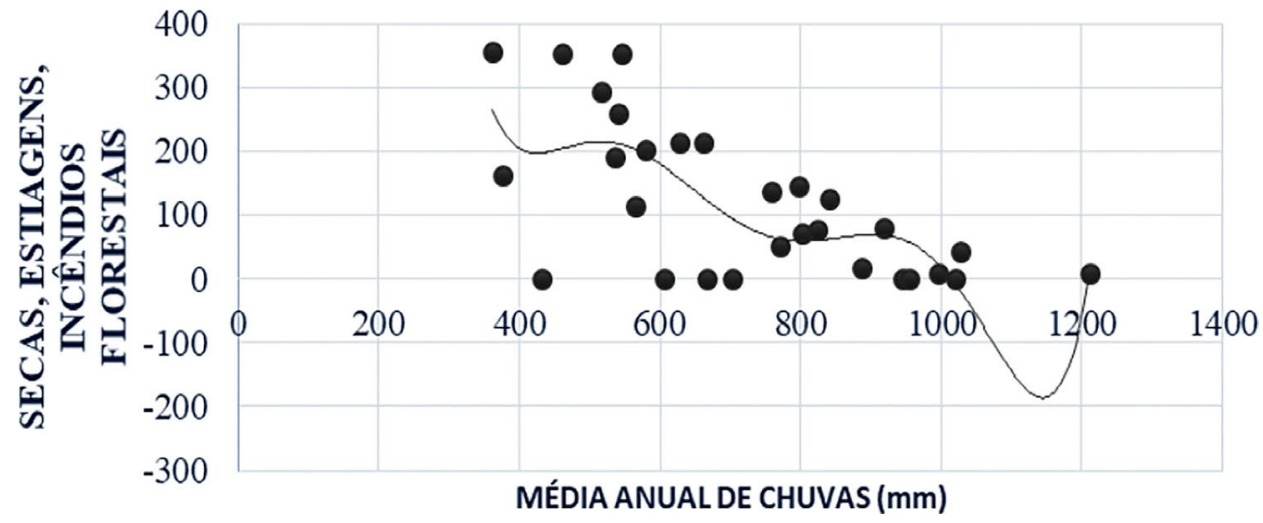

Figura 12 - Relação entre as chuvas e as secas/estiagens/incêndios florestais no Ceará (1991-2019). Fonte: Lima (2020).

chuvas intensas ao longo da série temporal da pesquisa, de 1991 a 2019, como mostra o Fig. 13.

Observa-se, de modo geral, que quando há picos de valores acumulados de chuvas, como em 2000, 2004, 2008 e 2009, tem-se um aumento dos registros de inundações, enxurradas, alagamentos e chuvas intensas. Já quando há valores acumulados de chuvas no ano, como em 1993, 1998, 2005, 2010 e 2012, assimila-se uma considerável diminuição do número de registros de tais desastres naturais.

Tabela 7 - Resultados da simulação dos dados da média mensal de chuvas e quantidade de desastres naturais registrados no Ceará de 1991 a 2019, no software Geogebra.

\begin{tabular}{lc}
\hline Variáveis & Resultados \\
\hline Média $(\mathrm{X})$ & 722,91 \\
Média $(\mathrm{Y})$ & 118,59 \\
Desvio padrão de X $\left(\mathrm{S}_{\mathrm{x}}\right)$ & 217,44 \\
Desvio padrão de $\mathrm{Y}\left(\mathrm{S}_{\mathrm{y}}\right)$ & 118,69 \\
Coeficiente de determinação $\left(\mathrm{R}^{2}\right)$ & 0,68008 \\
Coeficiente de correlação de Pearson $(\mathrm{r})$ & $-0,65597$ \\
Coeficiente de correlação, covariância $(\rho)$ & $-0,60078$ \\
SEQ & 126183 \\
\hline
\end{tabular}

Fonte: Lima (2020).
Foi feita a análise multivariada no software Geogebra, obtendo-se o diagrama de dispersão ilustrado na Fig. 14.

A Eq. (9) representa a equação de regressão não-linear (polinômio com equação de nono grau), das inundações, enxurradas, alagamentos e chuvas em função do volume de chuvas anuais, entre 1991 a 2019.

$$
\begin{gathered}
y=f(x)=-4,8929 \mathrm{E}-20 \times 9+3,3501 \mathrm{E}-16 \mathrm{x} 8- \\
1,0070 \mathrm{E}-12 \times 7-1,7435 \mathrm{E}-9 \times 6-1,9154 \mathrm{E}- \\
6 \times 5+1,3835 \mathrm{E}-3 \times 4-0,6565 \times 3+ \\
197,13 \times 2-339,38 \mathrm{x}+2549300
\end{gathered}
$$

A Tabela 8 mostra os resultados das variáveis estatísticas a partir da simulação no software Geogebra.

A interpretação dos resultados, a partir da simulação no software Geogebra, indica que o valor do coeficiente de determinação $\mathrm{R}^{2}$ é REPRESENTATIVO, demonstrando que o polinômio de regressão não-linear representou de forma válida os pontos de intersecção das variáveis analisadas e que o valor da correlação de Pearson apresentou um valor classificado como MODERADO e POSITIVO, revelando a $F O R C ̧ A$ da relação entre as duas variáveis ao 


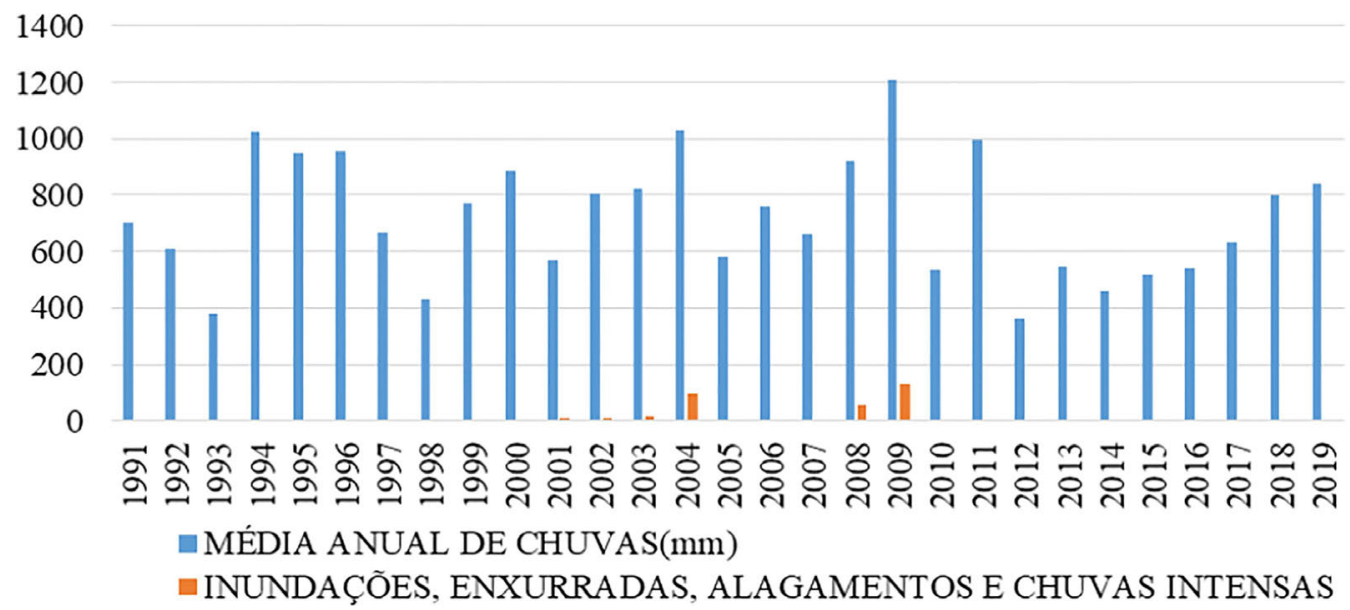

Figura 13 - Acumulado mensal de chuvas x desastres naturais no Ceará (1991-2019). Fonte: Lima (2020), Funceme (2020) e Cedec (2020).

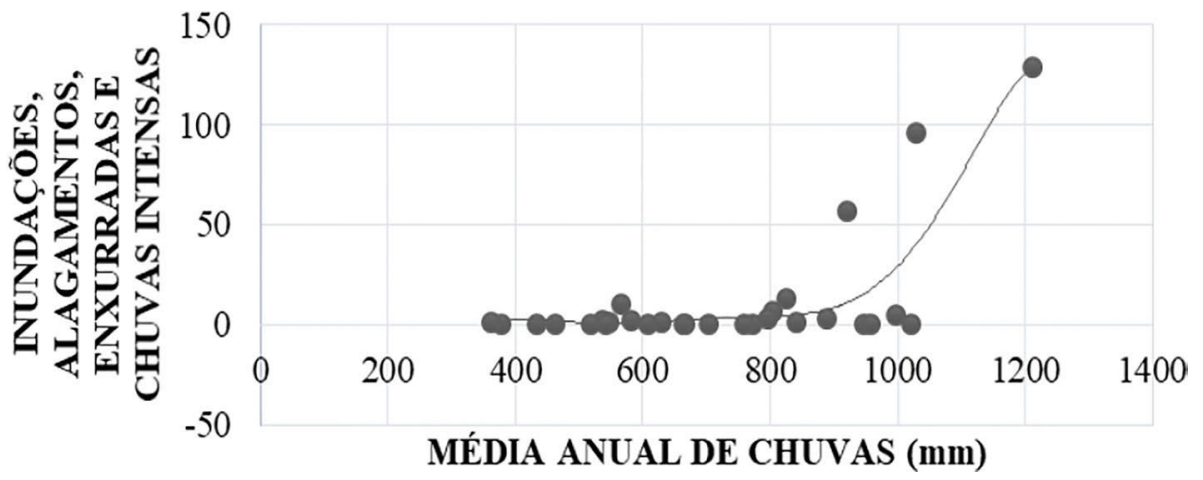

Figura 14 - Relação entre chuvas e inundações/enxurradas/alagamentos/chuvas intensas no Ceará (1991-2019). Fonte: Lima (2020).

Tabela 8 - Resultados da simulação dos dados da média mensal de chuvas e quantidade de desastres naturais registrados no Ceará de 1991 a 2019, no software Geogebra.

\begin{tabular}{ll}
\hline Variáveis & Resultados \\
\hline Média $(\mathrm{X})$ & 722,91 \\
Média $(\mathrm{Y})$ & 11414 \\
Desvio padrão de X $\left(\mathrm{S}_{\mathrm{x}}\right)$ & 217,44 \\
Desvio padrão de $\mathrm{Y}\left(\mathrm{S}_{\mathrm{y}}\right)$ & 30,297 \\
Coeficiente de determinação $\left(\mathrm{R}^{2}\right)$ & 0,77793 \\
Coeficiente de correlação de Pearson $(\mathrm{r})$ & 0,39666 \\
Coeficiente de correlação, covariância $(\rho)$ & 0,56802 \\
SEQ & 5707,3 \\
\hline
\end{tabular}

Fonte: Lima (2020).

nível de significância menor ou igual a 0,05 . Logo, as variáveis comportam-se na série temporal de 1991-2019 da seguinte forma: quanto maior o valor observado das chuvas acumuladas, maior o número de registros oficiais de inundações, enxurradas, alagamentos e chuvas intensas.

Entretanto, tanto secas, estiagens e incêndios florestais quanto inundações, enxurradas, alagamentos e chuvas intensas no Ceará podem relacionar-se com a ocorrência de fenômenos climáticos como os ENOS, em uma escala global maior, o El Niño, que pode influenciar em déficit de chuvas para o Estado, aumentando o número de registros de secas, estiagens e incêndios florestais.

Em anos de La Niña (fenômeno oposto ao El Niño), podem ocorrer chuvas abundantes no estado, principalmente no período que corresponde ao quadrimestre chuvoso, provocando inundações, enxurradas, alagamentos e chuvas intensas.

\section{Conclusões}

A publicação do Atlas Brasileiro de Desastres Naturais, com dados compilados do ano de 1991 a 2012, marcou o início do processo de avaliação e análise das séries históricas de desastres naturais no Brasil.

Para estado do Ceará, havia a necessidade não só de estudar os desastres naturais acometidos nessa região do semiárido, atualizando a série histórica para o intervalo de 1991 a 2019, como também incluir um embasamento quanto à meteorologia e a climatologia.

Dessa forma, a partir do problema da pesquisa, que perpassou a busca de resposta para a indagação sobre 
como os desastres naturais identificados no estado do Ceará estão relacionados à variabilidade climática, com destaque aos associados à precipitação, iniciou-se o desafio para obter tal resposta.

Nesse ensejo, assimilou-se que a variabilidade climática é uma característica associada, principalmente, ao regime de chuvas na região. Além disso, a análise dos sistemas atmosféricos e dos fenômenos climáticos permitiram um entendimento mais acurado e uma compreensão maior quanto às ameaças em relação aos desastres.

Assim, a pesquisa atingiu o primeiro de três objetivos específicos propostos, que foi analisar a variabilidade climática no estado do Ceará, nomeadamente quanto à precipitação associada aos sistemas atmosféricos e fenômenos climáticos, no período de 1991 a 2019.

De toda forma, apesar de determinados eventos provocarem impactos humanos, materiais e ambientais, que certamente indicariam a caracterização de um desastre, há uma subjetividade na missão de identificá-los ou não como desastres naturais.

Os maiores quantitativos de desastres naturais, mais de $90 \%$, são associados às secas e estiagens. Esses podem ser verificados com maior destaque nos Sertões Cearenses.

O segundo objetivo específico, de identificar e classificar os desastres naturais registrados no Estado do Ceará, por tipo, município e mesorregião, no período de 1991 a 2019, e representá-los por meio de tabelas, gráficos e mapas ilustrativos, foi igualmente atingido.

Atinou-se que o registro de desastres naturais segue uma sincronia com o fim da quadra chuvosa, no primeiro semestre de cada ano, com nova decretação no início das chuvas de pré-estação, no fim do segundo semestre de cada ano, indicando certo automatismo burocrático.

O terceiro e último objetivo específico foi igualmente atingido, afinal se estabeleceu a correlação estatística dos desastres naturais identificados no estado do Ceará com a variabilidade climática, principalmente quanto à precipitação, no período de 1991 a 2019.

Assim, foi possível relacionar a ocorrência de desastres naturais com a dinâmica climática. Ainda nesse âmago, houve uma correlação apontando que quanto maior o volume de chuvas anuais, menor o registro de secas, estiagens e incêndios florestais, e maiores os quantitativos de registros de inundações, enxurradas, alagamentos e chuvas intensas.

Dessa forma, o objetivo geral também foi atingido, porquanto se mostrou como os desastres naturais identificados no estado do Ceará relacionam-se à variabilidade climática, sublinhando-se os associados à precipitação, no período de 1991 a 2019.

Esse olhar com mais clareza para o passado torna-se condição sine qua non para os gestores fundamentarem suas análises e direcionarem as decisões políticas e técnicas da gestão de risco para a mitigação das atuais ocorrên- cias, vislumbrando uma adaptação aos cenários futuros, dadas as mudanças climáticas.

Foi possível compreender a variabilidade climática e sua ameaça quanto aos desastres nas diferentes regiões. Sugere-se, por oportuno, a alteração de alguns parâmetros de registros, bem como novas formas de atuação diante dessas ocorrências que tanto vulneram a sociedade, com ênfase na prevenção, a fim de contribuir para a construção de comunidades resilientes.

\section{Referências}

BARRY, R.G.; CHORLEY; R.J. Atmosfera, Tempo e Clima. 9. ed. Porto Alegre: Bookman, 2013.

BISQUERRA, R.; SARRIERA, J.C.; MARTÍNEZ, F. Introdução à Estatística: Enfoque Informático Com o Pacote Estatístico SPSS. Porto Alegre: Artmed, 2004. 254 p.

FUNCEME, Fundação Cearense de Meteorologia e Recursos Hídricos. Calendário das Chuvas no Estado do Ceará. Disponível em: http://funceme.br/app/calendario/produto/ ceara/media/anual. Acesso em: 18 jul. 2020.

GOERL, R.F.; KOBIYAMA, M. Considerações sobre as inundações no Brasil. In: Anais XVI Simpósio Brasileiro De Recursos Hídricos, Porto Alegre: ABRH, 2005.

IPCC. Climate Change 2014: Impacts, Adaptation, and Vulnerability. Part A: Global and Sectoral Aspects. Contribution of Working Group II to the Fifth Assessment Report of the Intergovernmental Panel on Climate Change FIELD, C.B.; BARROS, V.R.;DOKKEN, D.J.; MACH, K.J.; MASTRANDREA, M.D. et al. (eds.). Cambridge: Cambridge University Press, 1132 p., 2014.

LIMA, M.A.S. A Variabilidade Climática e os Desastres Naturais no Estado do Ceará (1991-2019). Dissertação de Mestrado Profissional em Climatologia e Aplicações nos Países da CPLP e África, Centro de Ciências e Tecnologia, Universidade Estadual do Ceará, Fortaleza, 2020.

MACHADO, V.S. Princípios de Climatologia e Hidrologia. Porto Alegre: SAGAH, 2017.

UNIVERSIDADE FEDERAL DE SANTA CATARINA. Atlas Brasileiro de Desastres Naturais 1991 a 2012: Volume Ceará. 2. ed. rev. ampl. Florianópolis: CEPED UFSC, 2013.

\section{Internet Resources}

CEDEC, Coordenadoria Estadual de Defesa Civil. Contatos e informações dos Órgãos Municipais de Proteção e Defesa Civil. Disponível em: http://www.defesacivil.ce.gov.br/ index.php?option $=$ com_content\&view $=$ article $\&$ id $=88$ : orgaos-municipais-de-protecao-e-defesa-civil\&catid $=14$ : lista-denoticias\&Itemid=81. Acesso em: 18 jul. 2020.

INMET, Instituto Nacional de Meteorologia. Disponível em: https://clima.inmet.gov.br/GraficosClimatologicos/DF/ 83377. Acesso em: 11 set. 2020.

License information: This is an open-access article distributed under the terms of the Creative Commons Attribution License (type CC-BY), which permits unrestricted use, distribution and reproduction in any medium, provided the original article is properly cited. 